\title{
Control Algorithm of Two-Phase Hybrid Stepper Motor Based on Three-State Hysteresis
}

\author{
Xiao Shenping, Wang Xiaolin \\ College of Automation, Nanjing University of Aeronautics \& Astronautics, Nanjing, China
}

Email address:

xiaosp@nuaa.edu.cn (Xiao Shenping),wangxl@nuaa.edu.cn (Wang Xiaolin)

\section{To cite this article:}

Xiao Shenping, Wang Xiaolin. Control Algorithm of Two-Phase Hybrid Stepper Motor Based on Three-State Hysteresis. Science Discovery. Vol. 6, No. 5, 2018, pp. 399-405. doi: 10.11648/j.sd.20180605.24

Received: September 30, 2018; Accepted: October 15, 2018; Published: October 18, 2018

\begin{abstract}
When the drive system of two-phase hybrid stepper motor adopts the traditional hysteresis loop control mode, it has good dynamic response performance, but also has some disadvantages such as big current ripple and big noise. In view of the above problems, the given current within one period of the winding is divided into ascending area, flat area and descending area, and the different demands for the rate of change of current in each area are analyzed in this paper. Compared with the traditional hysteresis loop, the three-state hysteresis loop has more switch combinations, which can meet the requirement of switching multiple current change rate in the driving system of two-phase hybrid stepping motor. This control algorithm based on three-state hysteresis loop presented in this paper can not only guarantee the dynamic response performance, but also greatly reduce the current ripple. The relationship between current ripple and terminal voltage, inductance and control frequency is derived in this paper. The hardware platform was set up with Cyclone IV FPGA of Altera company as the core processing chip, and the two-phase hybrid stepping motor of Oriental motor company model PK299DB was taken as the control object for experimental verification. Simulation and experiment verify the effectiveness of the algorithm.
\end{abstract}

Keywords: Two-phase Hybrid Stepping Motor, Current Ripple, Three-state Hysteresis Loop

\section{基于三态滞环的两相混合式步进电机控制算法}

肖申平, 王晓琳

自动化学院, 南京航空航天大学, 南京, 中国

\section{邮箱}

xiaosp@nuaa.edu.cn (肖申平), wangxl@nuaa.edu.cn (王晓琳)

摘要: 两相混合式步进电机驱动系统采用传统滞环控制方式时, 具有良好的动态响应性能, 但存在电流纹波大、噪声 大等缺点。针对上述问题，本文将绕组一个周期内的给定电流划分为上升区、平顶区和下降区，分析了各个区域对电 流变化速率的不同需求。相比传统滞环, 三态滞环的开关组合更多, 能够满足两相混合式步进电机驱动系统多种电流 变化速率切换的要求。本文提出的这种基于三态滞环的控制算法不仅能够保证动态响应性能, 而且极大的减小了电流 纹波。本文推导了电流纹波与端电压、电感、控制频率的关系式, 给出了算法中核心参数一一阀值的详细选取方法。 并以Altera公司Cyclone IV FPGA为核心处理芯片搭建了硬件平台, 以东方马达公司型号PK299DB两相混合式步进电机 为控制对象, 进行实验验证。仿真和实验都验证了该算法的有效性。

关键词：两相混合式步进电机，电流纹波，三态滞环 


\section{1. 引言}

步进电机由于其结构的特殊性, 能够将外部的脉冲信号 转化为电机转动的角度, 现在的技术已经能够做到每一次转 动角度的大小可以通过控制器任意控制。在步距角一定的情 况下, 电机转速完全正比于外部脉冲的频率, 电机转动角度 完全正比于接收的外部脉冲个数 [1]。正是这样的特点让混合 式步进电机采用开环控制而达到很高的控制精度。

相比于其他类型的电机, 步进电机更加适合开环定位 和低速下的开环调场合 [1-3]。而两相混合式步进电机出现 最早、控制最为成熟，是目前市场上应用最为广泛的步进 电机种类。主要应用与包装机、线切割的工作台拖动等工 业场合, 除了高精度的定位要求, 降低损耗也是重要的性 能指标, 这对厂家的成本控制有重要意义。另外在打印机、 喷绘机、写真机等办公场合也有其广泛的应用, 因此运行 平稳、响应快、降低噪声也是衡量步进电机驱动系统重要 的性能指标。综上, 提高两相混合式步进电机驱动系统的 动态特性、降低损耗越来越成为研究的热点。

目前学者主要对两相混合式步进电机的低频振荡的 抑制和提高动态性能进行了研究。解决的思路可以总结为 四个方面: 优化电机结构、改善驱动方式、优化加减速曲 线和提升控制芯片性能[4-7]。其中应用最为广泛的驱动方 式是电流闭环驱动, 即将实际绕组电流实时控制, 让其能 够跟随给定, 从而达到控制转矩的目的, 进而控制转速、 转向等。因此电流纹波和给随给定的快速性成为驱动系统 重要的指标。为了优化这两个指标, 众多学者进行了研究。 常见的电流闭环控制策略有滞环、PID、模糊控制等方式 来实现PWM的斩波控制 [9-11]。作为最为直接的控制方式, 滞环的优缺点都很鲜明: 优良的动态性能和较大的纹波。 在滞环控制方式下对纹波的抑制主要有两点: 减小算法午 杂度、提高芯片运算能力等来减小控制周期和灵活选择绕 组续流通路。但不同续流方式对两相混合式步进电机绕组 电流纹波的影响任然没有系统的理论研究, 且整个周期内 续流方式的分配也有待进一步的研究。文献[9]提出根据接 收脉冲频率的大小自动调整快慢续流时间的控制方式,但 是没有给出高频、低频和正常频率的划分标准和理论依据。 文献[10]、[11]研究了混合使用续流方式, 给出的分配方 式是将一个周期平均划分然后顺序选用不同的续流方式, 是否是最优的分配方式没有给出依据。本文对最优的续流 分配方式进行了研究, 并给出理论说明。

本文推导了电流纹波表达式, 给出了电流纹波与端电 压、反电势、电感、电阻和控制频率的关系式。并分析了 端电压与开关管开通状态的关系，从而提出了一种基于三 态滞环的两相混合式步进电机的控制算法, 给出了核心参 数阀值 $\mathrm{H}$ 的选取依据。该算法能有效的减小电流纹波, 降 低开关损耗和电流THD, 减小振动和噪声。并通过仿真和 实验验证了算法的正确性。

\section{2. 基于三态滞环的控制算法}

两相混合式步进电机绕组给定电流不同时段需要不 同的电流变化速率, 而最常采用的电路拓扑是 $\mathrm{H}$ 桥结构, 本文将电力电子中三态滞环的概念引入电机控制, 相比于
传统滞环控制, 三态滞环多了 0 态, 对应到系统中即多了 一种续流回路, 也即多了一种电流变化速率, 灵活使用三 态对电机进行控制能用发挥它们各自的优势, 从而提升电 机驱动系统的性能。

\section{1. 给定电流区域划分}

\subsection{1. 给定电流的确定}

两相混合式步进电机每相绕组单独作用, 分别正负通 电时, 即按照 $\mathrm{A}+\mathrm{B}-、 \mathrm{~A}+\mathrm{B}-$ 的通电顺序通电时会产生 4 个方向的转矩如图所示:

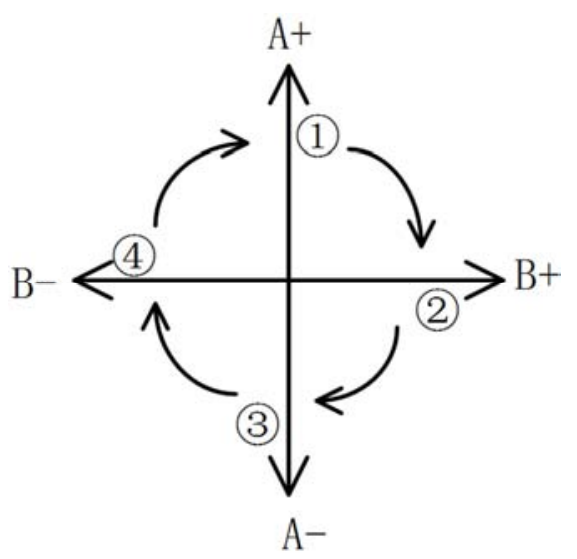

图1 两相混合式步进电机转矩示意图。

上述的通电逻辑即为两相混合式步进电机最为基础 的整步运行, 在这种运行模式下, A、B相绕组的给定电 流如下图所示:

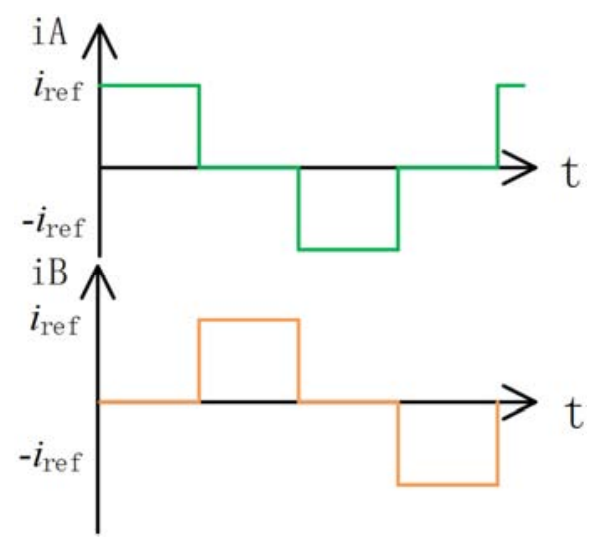

图2 整步模式下A、B相绕组给定电流波形。

整步运行模式下通电逻辑简单, $\mathrm{AB}$ 相绕组的电流给 定值为矩形波, 但这种运行模式下步距角较大, 运行过程 中噪声和振动都较大。

为了抑制低频振荡和减小噪声, 两相混合式步进电机 驱动系统多采用细分驱动的方式。所谓的细分驱动是指将 传统的电流矩形波分解为阶梯波, 从而将初始步距角变为 任意步距角。将步距角分解的原理如下图所示: 


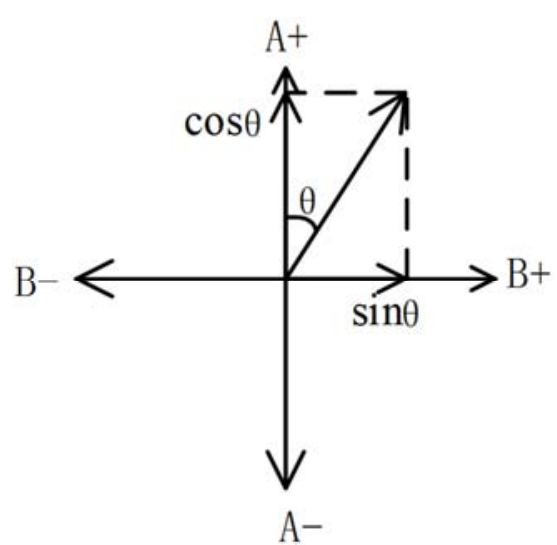

图3 微分模式下A、B相绕组给定电流分解示意图。

在步距角无限小时，A、B相绕组给定电流波形是幅 值相等、相位相差 $90^{\circ}$ 的正弦波形。考虑到核心处理芯片 的运算能力和开关器件最大动作频率的限制, 步距角实际 上不能做到无限小, 而是阶梯波。以4细分为例, A、B相 绕组电流给定波形如下:

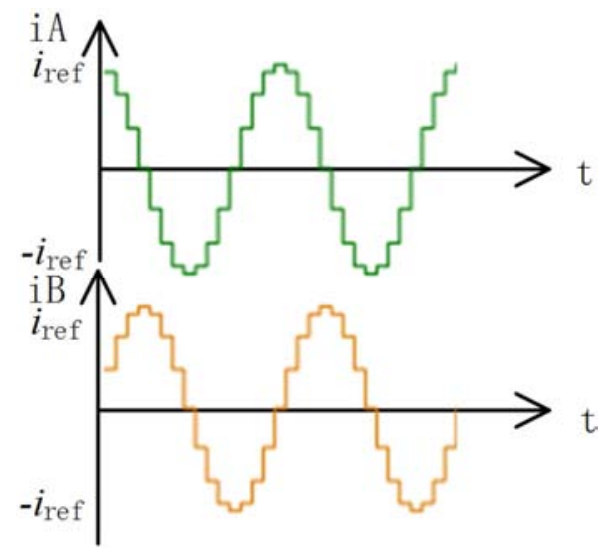

图4 A、B相绕组给定电流波形。

\subsection{2. 给定电流的划分}

不难发现绕组给定电流是无限接近正弦波的阶梯波, 可以划分为三个区域, 分别为电流突升的上升区、电流保 持恒定的平顶区和电流突降的下降区。

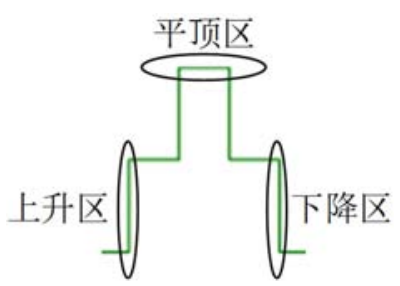

图5 绕组给定电流区域划分。

这样划分区域的目的在于明确给定电流各个阶段对 电流变化率的需求。在上升区和下降区, 给定电流都有一 个突变的过程, 为了能够让实际电流跟随给定, 这两个阶 段需要较大的电流变化率, 从而保证系统的快速性。而在 平顶区, 给定电流保持不变, 实际电流在稳定在给定值左
右时, 需要相对较小的电流变化率, 否则在相同的控制周 期内, 实际电流会与给定偏差过大，造成电流纹波过大。 划分区域后, 一个周期内绕组电流需要的电流变化率 可以定量确定。其结果可以用下表表示:

表1 各区域电流变化率需求。

\begin{tabular}{llll}
\hline 给定电流区域 & 上升区 & 平顶区 & 下降区 \\
\hline 电流变化率需求 & 快速上升 & 缓慢变化 & 快速下降 \\
\hline
\end{tabular}

具体实现不同的电流变化率需要功率器件不同的开 关组合, 不同开关组合与不同速率的对应关系将在下文详 细介绍。

\section{2. 三态滞环控制策略}

两相混合式步进电机的A、B相绕组采用开绕组的形 式, 空间上相差 $90^{\circ}$, 互感影响可以忽略不计, 因此A、B 相可以看做相互独立, 互不影响[7]。在主电路采用 $\mathrm{H}$ 桥结 构的时候, 每相绕组的电流也能够单独控制, 故仅以 $\mathrm{A}$ 相 绕组为例, 分析绕组电流电流闭环控制原理, B相绕组的 电流控制与 $\mathrm{A}$ 相同理。采用 $\mathrm{H}$ 桥主电路时 $\mathrm{A}$ 相绕组的控制拓 扑如下图所示:

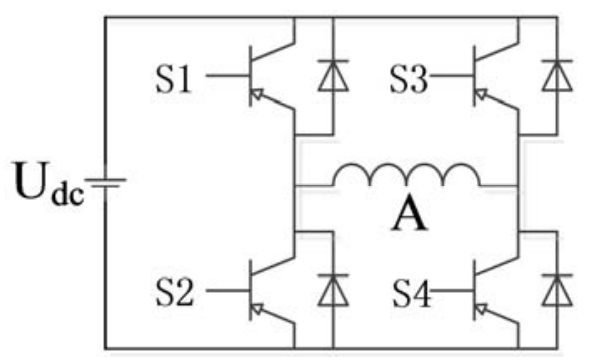

图6 H桥主电路。

A相绕组的电流给定值由环形分配器在脉冲的作用下 实时输出, 电流的实际值由电流传感器读出。当 $\mathrm{A}$ 相绕组 的电流需要正向增大的时候, 只有一种开关管的组合, 即 开通1、4管, 关闭2、3管, 此时的A相绕组两端施加了正 电压, 电感电流正向增大。A相绕组电流正向增大的电流 走向如下图所示:

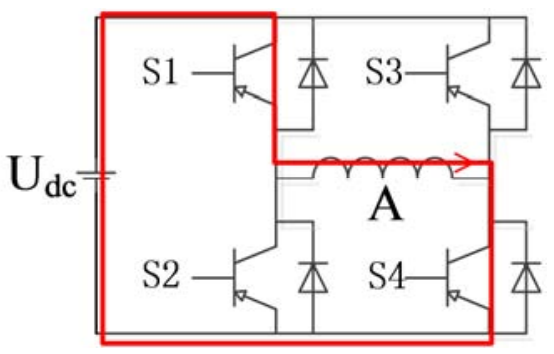

图7 A相绕组正向增大电流示意图。

当 $\mathrm{A}$ 相绕组的电流需要减小的时候, 常用的开关管组 合有两种：只导通2、3管或者只导通2、4管，这两种组合 下的电流方向并不相同, 加在绕组上的电压也不相同, 因 此在这两种开关组合下, 绕组的电流变化速率不相等。

在只导通2、3管的情况下，电流流向如下图所示: 


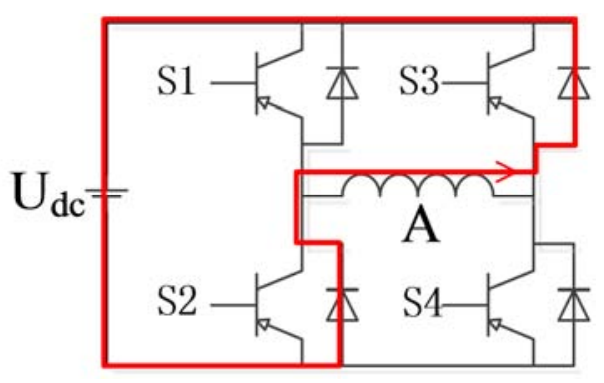

图8 导通2、3管电流示意图。

电感上的电流不能突变, 因此绕组上的电流方向不变 通过2、3管的续流二极管流经电源形成回路。而在只导通 2、4管的情况下，电流沿4管、2管形成回路，如下图所示:

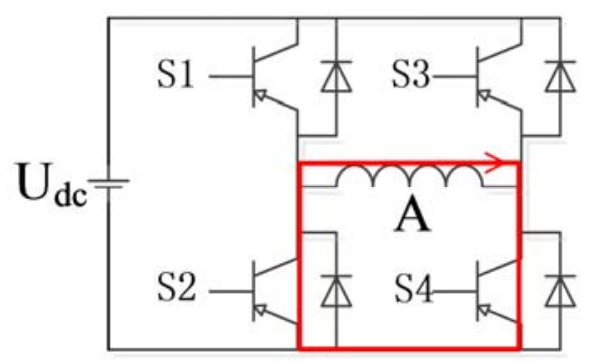

图9 导通2、4管电流示意图。

为了找出两种续流回路电流变化率的具体数值, 需要 列写回路的电压方程。首先电感两端的电压与流经电感的 电流关系为:

$$
L \frac{\mathrm{d} i}{\mathrm{~d} t}=u
$$
列写为:

则两相混合式步进电机A相绕组的基本电路方程可以

$$
u_{A}=R i_{A}+L \frac{d i_{A}}{d t}+e
$$

其中:

$L$ 为绕组电感值;

$i_{A}$ 为 $\mathrm{A}$ 相绕组电流;

$u_{A}$ 为绕组电感两端的压降;

$R$ 为绕组上的压降;

$e$ 为反电势;

解此微分方程可以得到 $\mathrm{A}$ 相电流随时间变化的表达式 如下:

$$
i_{A}=\frac{u_{A}-e}{R}+C_{l} e^{-\frac{R}{L} t}
$$

其中常数 $C_{1}$ 与 $\mathrm{A}$ 相绕组的初始状态有关, 选择一个控 制周期的起始时间为 $\mathrm{t}=0$, 此时 $\mathrm{A}$ 相绕组的初始电流为 $i_{0}$, 代 入上式可以求得:

$$
C_{1}=i_{0}-\frac{u_{A}-e}{R}
$$

因此在一个控制周期内电流的改变量就是 $\mathrm{t}=\mathrm{T}$ 时刻的 电流值与 $i_{0}$ 的差值, 即:

$$
\Delta i=\left(1-e^{-\frac{R}{L} T}\right)\left(\frac{u_{A}-e}{R}-i_{0}\right)
$$

在电机稳态运行的情况下, 转速一定, 所以反电势也 是常数, 因此每个控制周期内电机绕组电流的变化量与加 在电机绕组的端电压正相关。在图8中, 绕组电感两端施 加了反向母线电压, 电流迅速衰减; 在图9中, 绕组电感 两端电压为 0 , 绕组电流以续流的方式缓慢衰减 $[8]$ 。在电 流需要减小的情况下, 采用两种开关管的导通方式会得到 两种不同下降速度的结果, 一个周期内电流的下降量可由 上式得到。这两种特性的电流下降方式各有利弊, 而在步 进电机的微分驱动系统中, 混合的使用者两种方式才能得 到最佳的控制性能 $[9,10]$, 具体分析如下。

在微分运行的时候, 每相的给定电流波形是一个阶梯 波, 可以分为两个部分: 平顶部分和电流突降部分。前者 电流基本接近给定, 采用缓慢续流的方式, 能够在电流低 于给定的第一时间发现，并在该周期将电流拉高。这种情 况下, 电流的纹波得到了有效减小; 后者电流与电流给定 相差很大, 即电流需要突降, 只有采用快速衰减的方式, 才能做到电流迅速给谁给定, 保证驱动系统的动态性能。

上述的控制思想可以总结为: 电流需要减小的时候, 若实际电流与给定电流相差很小, 则采用缓慢续流, 若相 差很大, 则采用快速衰减的方式。具体实现即采用三态滞 环 [8]: 选定一个电流给定值与实际值相差的标准值, 称为 阀值 $\mathrm{H}$, 当 $\left|i_{r e f}-i_{f b}\right|>\mathrm{H}$ 时, 只导通 23 管为 -1 态; 当 $\left|i_{r e f}-i_{f b}\right|<\mathrm{H}$ 时只导通 24 管为 0 态; 当 $i_{r e f}-i_{f b}<0$ 时, 电流 需要正向增大, 只导通 14 管为 +1 态不需要讨论。

\section{3. 阀值 $\mathbf{H}$ 的选取}

差值标准 $\mathrm{H}$ 的选取实际上代表了电流实际值与给定值 相差的程度。超过 $\mathrm{H}$ 说明电流实际值与给定值相差过大, 不超过 $\mathrm{H}$ 说明电流实际值与给定值相差不大。记电流快速 衰减时一个控制周期下降量为 $\mathrm{M}$, 缓慢续流一个控制周期 电流的下降量为 $\mathrm{m}$, 从上一节推导可知:

$$
\left\{\begin{array}{l}
M=\left(1-\mathrm{e}^{-\frac{R}{L} T}\right)\left(\frac{\mathrm{u}_{d c}+\mathrm{e}}{R}+i_{0}\right) \\
m=\left(1-\mathrm{e}^{-\frac{R}{L} T}\right)\left(\frac{\mathrm{e}}{R}+i_{0}\right)
\end{array}\right.
$$

在最理想的情况下, 实际电流一旦低于给定值就立刻 被检测出来, 并开通 $1 、 4$ 管让电流上升, 经过一个周期 $\mathrm{T}$, 电流的上升量为: 


$$
\Delta i=\left(1-e^{-\frac{R}{L} T}\right)\left(\frac{u_{A}-e}{R}-i_{0}\right)
$$

这个上升量是电流实际值接近电流给定值时可以接 受的最大差值, 只要差值小于该值都认为电流实际值已经 什么接近给定了, 采用缓慢续流不影响系统的动态响应; 大于该值说明此时的电流实际还不够接近给定, 需要采用 快速衰减的开通方式来加快电流的给定, 因此该上升量就 是H值。

上式定义的 $\mathrm{H}$ 值并不是一个定值, 而是与初始的电流 值、转速有关的量。如果希望在保证系统动态性能的基础
上尽量减小电流纹波, 那么 $\mathrm{H}$ 的选值可以略小, 电流初始 值取系统允许通过的最大值。在实际应用的时候可以先取 $\mathrm{H}$ 的值为 0 , 观测电流波形一个控制周期的上升量, 再修改 $\mathrm{H}$ 的值与之相等即可。

\section{4. 仿真与实验结果}

选择采样频率 $50 \mathrm{KHz}$, 对电感做定电流 $2 \mathrm{~A}$ 定电流闭环 仿真实验, 在-1态和0态下电流波形如下图所示:
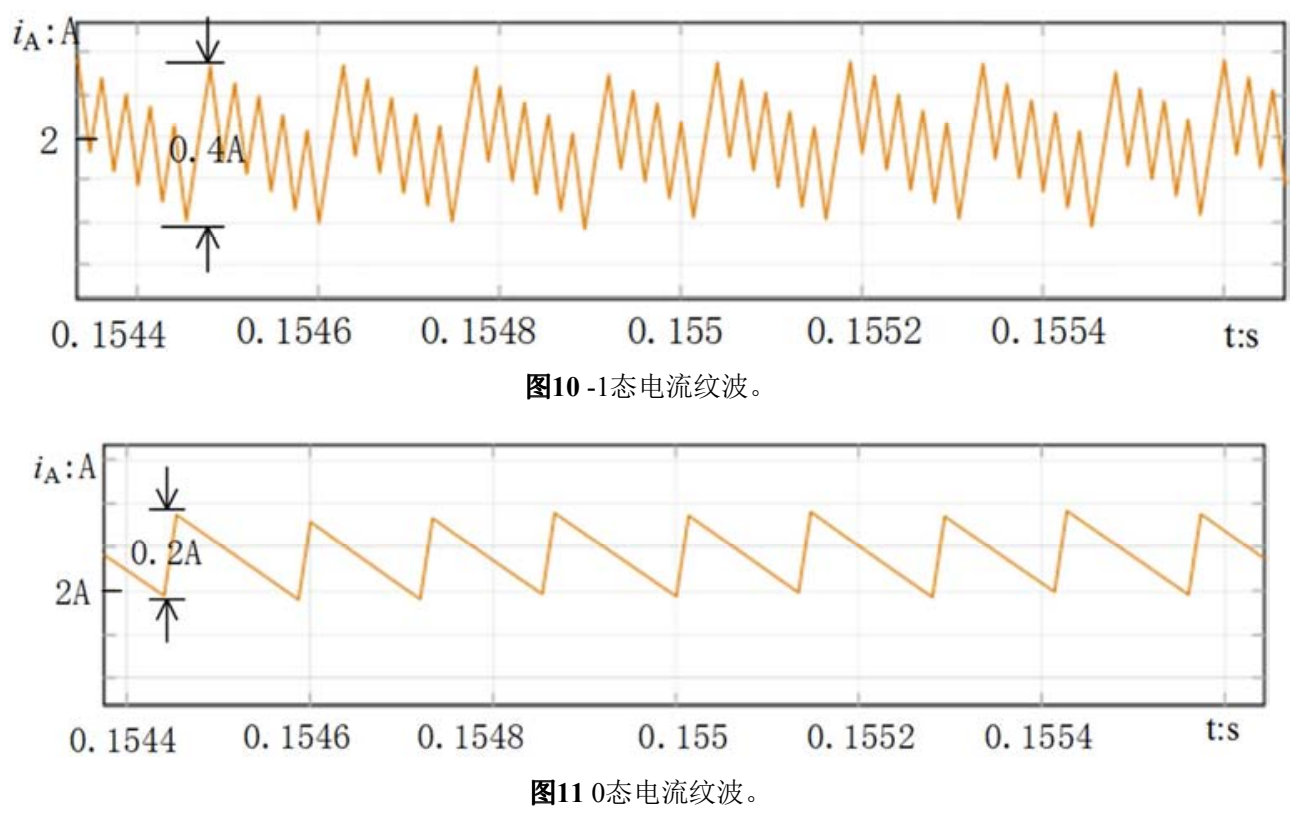

从仿真波形可以看出两种状态下的电流上升速率几 乎一样, 但下降速率相差很大。

在实际电流大于给定电流需要减小时, -1 态电流下降 速率很快, 在一个控制周期里相对来说减小的电流量更大, 也就是说实际电流在给定电流下面的纹波要更大; 而 0 态 电流由于下降速率慢, 能够在下降到给定电流的第一时间 被检测出来, 即实际电流在给定电流下面的纹波更小。

给定电流上面的纹波两者相差不大, 所以总体来说, 0 态的时候电流纹波大大减小了。另外从电流波形与电流 给定值的交截次数可以观测开关管的开通次数。明显在相 同的时间内, -1 态的电流波形与 $2 \mathrm{~A}$ 交截次数远远大于 0 态, 因此开关损耗得到了抑制。

分别采用全程-1态和混合使用三态进行仿真实验, 选 取A相的电流波形, 并对其做FFT分析, 在传统滞环的控 制算法下的结果如下:

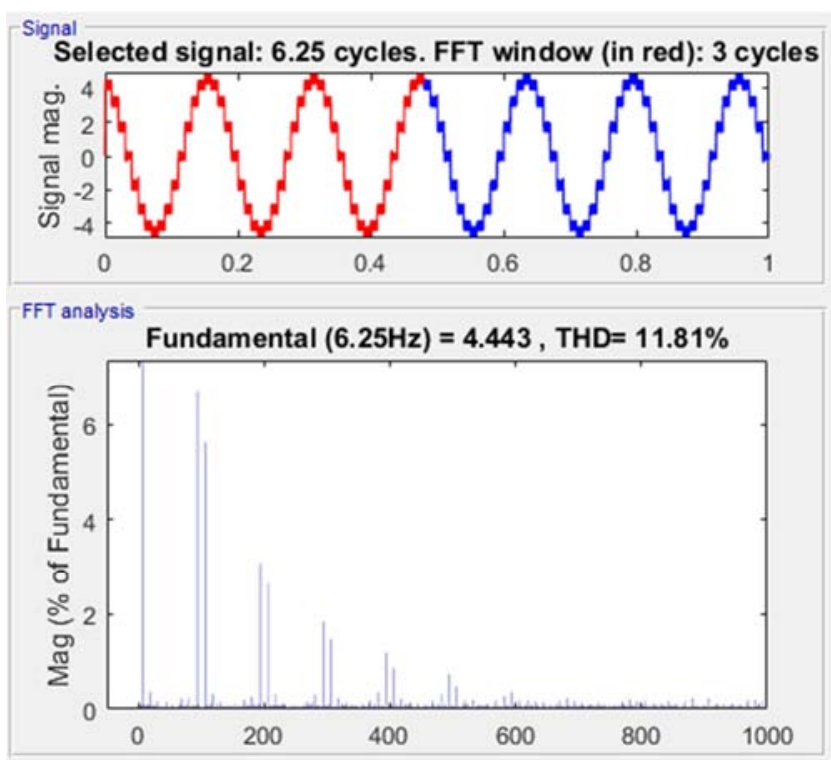

图12 快衰减电流模式。

在采用基于三态滞环的控制算法下的结果如下: 

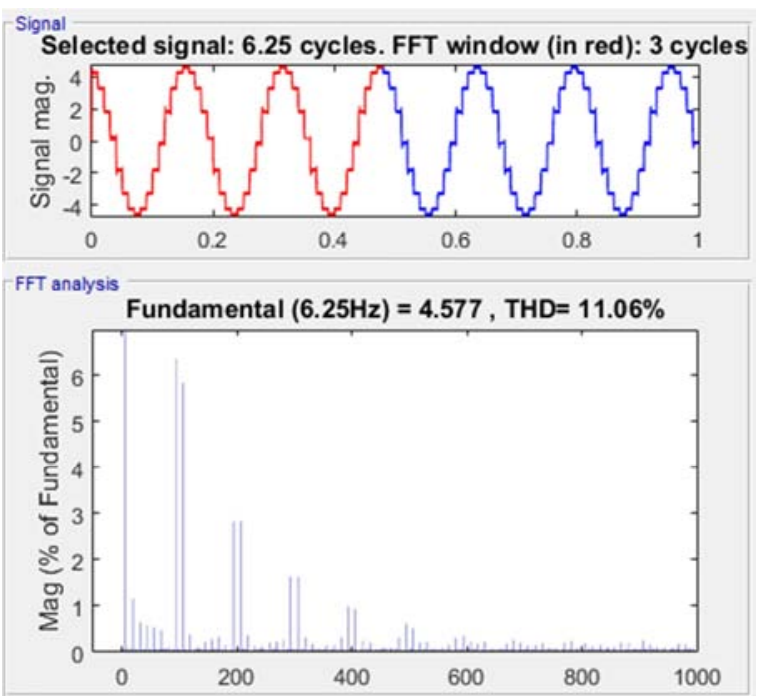

图13 混合衰减电流模式。

与传统的滞环相比, 采用三态滞环的控制方式也能够 很好的控制住电流, 并且从FFT分析中可以看出, 采用三 态滞环将THD从 $11.8 \%$ 降低到了 $11.06 \%$ 。

三态滞环控制中H值的选取至关重要, 上节给出了一 种理论上的值, 即为电感在一个控制周期内的上升量, 针 对 $\mathrm{H}$ 的变化对电流纹波的影响, 做了如下的仿真研究:

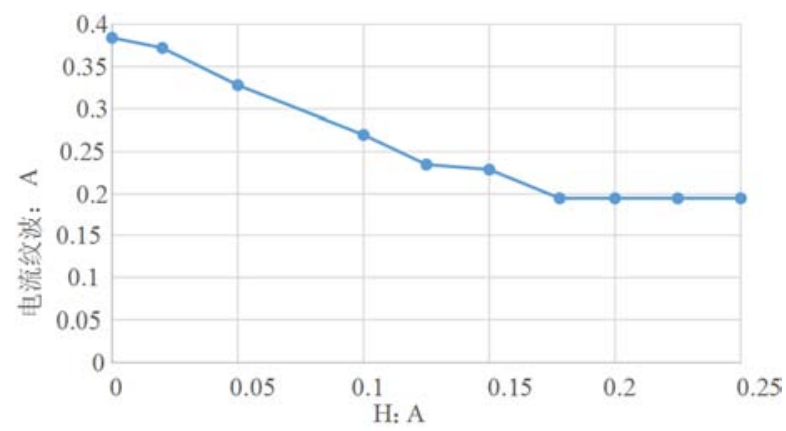

图14 不同H值对应绕组电流纹波图。
H选值越小, 采用-1态的概率就越高, 位于电流给定 值下部的电流纹波就相对较大; $\mathrm{H}$ 选值越大, 采用 0 态的概 率就越高, 位于电流给定值下部的电流纹波就越小.从折 线图可以发现当 $\mathrm{H}$ 的值接近 0 的时候, 此时的控制方式就是 普通的滞环, 电流纹波是最大的, 整体的电流纹波为一个 控制周期电流的上升量的两倍; 当 $\mathrm{H}$ 值逐渐增大的时候, 电流纹波也逐渐减小, 直到达到一个临界点就不再减小, 仿真验证该临界点就是理论值 $\mathrm{H}$, 即一个控制周期电流的 上升量。

为了验证上述算法进行了硬件实验验证。硬件平台以 Altera公司Cyclone IV FPGA为核心处理芯片, 以东方马达 公司型号PK299DB两相混合式步进电机为控制对象。硬件 平台图如下:

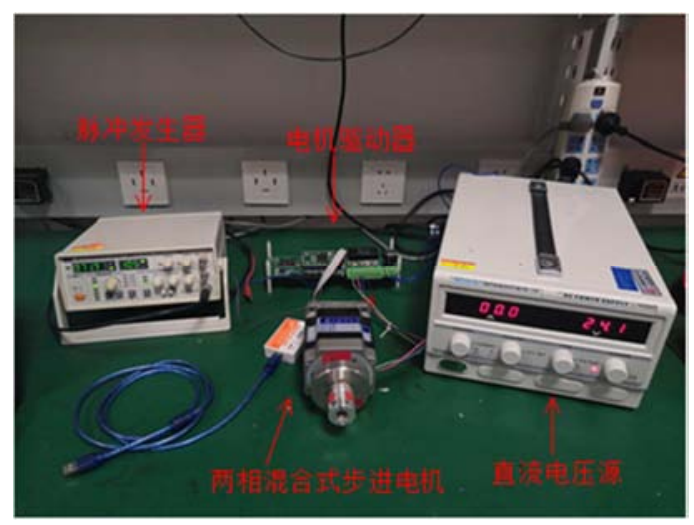

图15 硬件平台实物图。

在电流采样频率 $50 \mathrm{KHz}$, 脉冲频率 $50 \mathrm{~Hz}$, 电流幅值 $2 \mathrm{~A}$, 4细分的运行模式下, 两相混合式步进电机的A相电流波形 如图所示:

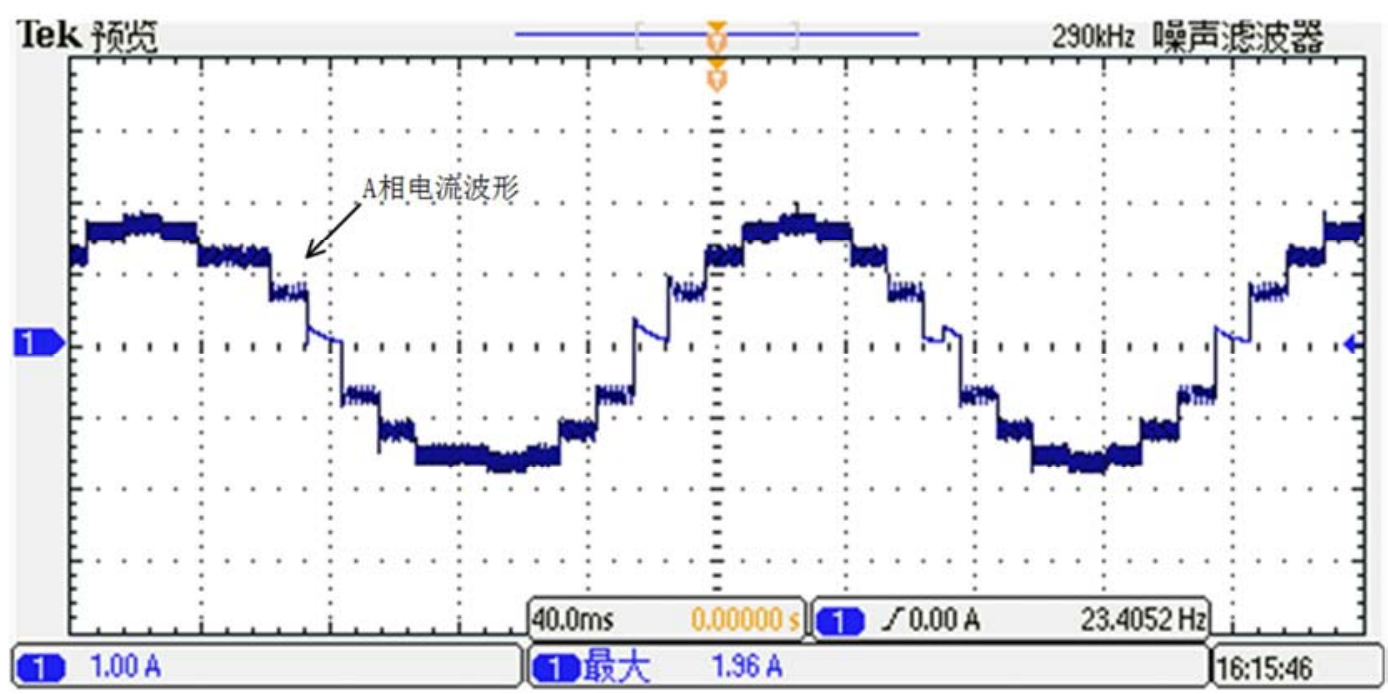

图16 A相绕组电流电流波形。 


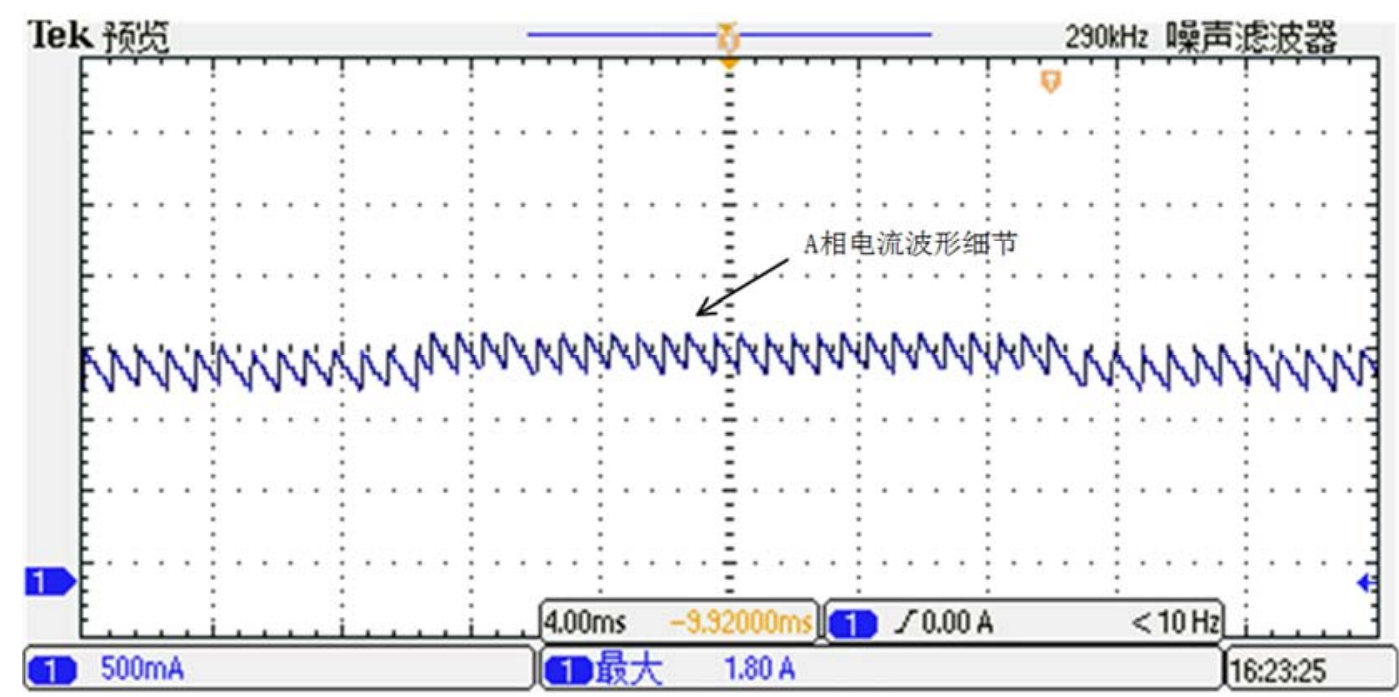

图17 A相绕组电流波形细节放大图。

在4细分的运行模式下，一个周期有 16 步，从实验波 形可以看出 $\mathrm{A}$ 相绕组电流波形为阶梯波, 接近正弦, 在一 个周期里有 16 个阶梯, 这与设定的运行模式相符。在每一 个阶梯的保持阶段, 电流基本稳定, 实际电流与给定相差 不大, 两者的差值不会超过标准值 $\mathrm{H}$, 采用 0 态优势较大; 而在阶梯下降阶段, 两者的差值会超过标准值 $\mathrm{H}$, 采用 -1 态能够快速跟随给定。整个阶段电流既能快速跟随给定, 又能减小电流纹波。

\section{5. 结论}

本文提出了一种基于三态滞环的两相混合式步进电 机的控制算法, 在电流需要减小时候, 通过电流实际值与 给定值的相差程度选择不同电流衰减方式, 并给出了核心 参数阀值 $\mathrm{H}$ 的选取依据, 从而得到续流方式的最优分配策 略。仿真与实验证明能够该控制算法能够有效降低电流纹 波, 一定程度上降低电流THD; 同时开关管的开通次数也 相比传统滞环控制减少，开关损耗得到了降低。

\section{参考文献}

[1] Dong N, Ming Z, Wen T. Two-Phase SVPWM Modulation Method and Its Application in Stepper Motor [C]// Tenth International Conference on Computational Intelligence and Security. IEEE Computer Society, 2014: 794-797.
[2]史敬灼,徐殿国,王宗培.混合式步进电动机伺服系统研究 [J]. 电工技术学报, 2006, 21 (4): 72-78。

[3] 聂巍. 步进电动机闭环控制系统研究[D].武汉理工大学, 2014。

[4] 高云广, 刘爱萍, 卜庆华.直线步进电动机闭环控制系统研究 [J].微电机, 2007,40 (6): 39-41。

[5] 瞧亮.两相混合式步进电机的新型控制策略研究[D].浙江大 学, 2011。

[6] Mihalache G, Zbant A, Livint G. Open-loop control of hybrid stepper motor with two phases using voltage to frequency converter [M]. 2013.

[7] 汪全伍.两相混合式步进电机高性能闭环驱动系统研究[D]. 浙江理工大学, 2016。

[8] 李姣丽,秦海鸿,邓翔, 等.三态滞环电流控制全桥逆变器建模 及控制 [J].电力电子技术, 2011, 45 (10): 89-91。

[9] Wang S, Liu J. Research on phase current and bus current of two-phase hybrid stepping motor and control system [C]// International Conference on Electrical Machines and Systems. IEEE, 2011: 1-4.

[10] 张航鲜.新型步进电机驱动电路的研制 $[D]$.西安电子科技大 学, 2007 。 